\title{
Epidemiology and direct medical costs of osteoporotic fractures in men and women in Switzerland
}

\author{
Kurt Lippuner · Matthias Golder · Roger Greiner
}

Received: 4 June 2004 / Accepted: 9 June 2004 / Published online: 16 September 2004

(C) International Osteoporosis Foundation and National Osteoporosis Foundation 2004

\begin{abstract}
The aim of this study was to estimate the hospitalization incidence and the total number of hospital days related to all fractures and osteoporotic fractures in the year 2000 in Switzerland and to compare these with data from other frequent disorders in men and women. The official administrative and medical statistics database of the Swiss Federal Office of Statistics (SFOS) from the year 2000 was used. It covered $81.2 \%$ of all registered patient admissions and was considered to be representative of the entire population. We included the ICD-10 codes of 84 diagnoses that were compatible with an underlying osteoporosis and applied the best matching age-specific osteoporosis attribution rates published for the ICD-9 diagnosis codes to the individual ICD-10 codes. To preserve comparability with previously published data from 1992, we grouped the data related to the ICD-10 fracture codes into seven diagnosis pools (fractures of the axial skeleton, fractures of the proximal upper limbs, fractures of the distal upper limbs, fractures of the proximal lower limbs, fractures of the distal lower limbs, multiple fractures, and osteoporosis) and analyzed them separately for women and men by age group. Incidences of hospitalization due to fractures were calculated, and the direct medical costs related to hospitalization were estimated. In addition, we compared the results with those from chronic pulmonary obstructive disease (COPD), stroke, acute myocardial infarction, heart failure, diabetes and breast carcinoma from the same database. In Switzerland during 2000, 62,535 hospitalizations for fractures $(35,586$ women and 26,949 men) were registered. Fifty-one percent of all
\end{abstract}

K. Lippuner $(\square) \cdot$ M. Golder

Osteoporosis Policlinic, University Hospital of Berne,

3010, Berne, Switzerland

E-mail: kurt.lippuner@insel.ch

Tel.: + 41-31-6323185

Fax: +41-31-6329596

R. Greiner

HealthEcon AG, Basle, Switzerland fractures in women and $24 \%$ in men were considered as osteoporotic. The overall incidences of hospitalization due to fractures were 969 and 768 per 100,000 in women and men, respectively. The hospitalization incidences for fractures of the proximal lower limbs and the axial skeleton increased exponentially after the age of 65 years. The direct medical cost of hospitalization of patients with osteoporosis and/or related fractures was 357 million CHF. Hip fractures accounted for approximately half of these costs in women and men. Among other common diseases in women and men, osteoporosis ranked number 1 in women and number 2 (behind COPD) in men. When compared with data from 1992, the average length of stay had shortened by 8.4 days for women and 4.7 days for men, leading to a decrease of almost $40 \%$ in direct medical costs related to acute hospitalizations. This apparent decrease in cost might result from a shift into the ambulatory cost segment, for which the assessment and management tools need to be developed. We conclude that, in 2000, osteoporosis continued to be a heavy burden on the Swiss healthcare system. Lack of awareness of the disease and its consequences prevents widespread use of drugs with antifracture efficacy. This limits their potential to reduce costs.

Keywords Costs $\cdot$ Epidemiology $\cdot$ Fractures · Hospitalizations $\cdot$ Men $\cdot$ Osteoporosis

\section{Introduction}

Osteoporosis is a skeletal disease characterized by low bone mass and a deterioration in bone micro-architecture that results in enhanced bone fragility and increased susceptibility to fractures [1]. The commonly reported osteoporotic fractures occur in the forearm, vertebra and hip [2]. However, osteoporosis is a systemic disease of the skeleton, and osteoporotic fractures may also occur at the proximal humerus, ribs, tibia, pelvis, knee, 
and ankle $[1,3,4,5,6]$. In osteoporotic patients virtually any bone may be fractured by so-called inadequate or low-energy trauma.

In 1992 we estimated the annual direct medical costs of hospitalization due to osteoporotic fractures in Switzerland that had been recorded by the medical statistics department of VESKA (Vereinigung Schweizerischer Krankenhaeuser, the Swiss Hospital Association), and compared these costs with those due to other common diseases in men and women. We concluded that osteoporotic fractures in women consumed more healthcare resources than chronic pulmonary obstructive disease (COPD), stroke, breast carcinoma and myocardial infarction (MI). In men, osteoporosis ranked fourth behind COPD, MI and stroke [7].

The aim of our study was to update our knowledge of the socio-economic burden of osteoporosis in Switzerland by analyzing the hospitalization incidence and the total number of hospitalization days related to osteoporotic fractures, and fractures in general, and to compare the total hospitalization days due to osteoporosis with those caused by other common disorders in men and women.

\section{Materials and methods}

Source of hospital statistics

The administrative and medical statistics database of the Swiss Federal Office of Statistics (SFOS) from 2000 was used. Since 1998 all Swiss acute hospitals, as well as rehabilitation centers and specialized clinics, have been obliged to supply data to the SFOS. There were 376 such institutions, and administrative statistics were available from 372 of them (four had closed during 2000). These administrative statistics consolidate the data relating to finance, human resources, numbers of beds and patient admissions. Medical statistics were available from 343 hospitals $(91.2 \%)$, corresponding to $81.2 \%$ of all registered patient admissions. We used those medical statistics to determine the number of hospitalized fractures and to calculate the mean length of stay (LOS) by fracture type, age group and gender. All data were extrapolated to $100 \%$ to become representative for Switzerland as a whole.
Definition of fracture and osteoporotic fracture

In the medical statistics database the diagnoses were coded as main or secondary. To calculate the numbers of diagnoses related to fractures and/or osteoporosis, we retained both main and secondary diagnoses. All diagnoses were coded according to the ICD-10 classification. Eighty-four ICD-10 codes were relevant to our analysis, and they were assigned to four categories:

- Category 1: osteoporosis coded as such. There were 21 relevant ICD-10 codes; osteoporosis without pathologic fracture: M81.0 (postmenopausal osteoporosis), M81.1 (osteoporosis after ovariectomy), M81.2 (immobilization osteoporosis), M81.3 (osteoporosis consecutive to post-surgical malabsorption), M81.4 (drug-induced osteoporosis), M81.5 (idiopathic osteoporosis), M81.6 (regional osteoporosis [Lequesne]), M81.8 (other osteoporosis), M81.9 (osteoporosis, no further mention), M82.0 (osteoporosis with plasmacytoma), M82.1 (osteoporosis with endocrine diseases), M82.8 (osteoporosis with other classified diseases); osteoporosis with pathologic fracture: M80.0 (postmenopausal osteoporosis with pathologic fracture), M80.1 (osteoporosis with pathologic fracture after ovariectomy), M80.2 (immobilization osteoporosis with pathologic fracture), M80.3 (osteoporosis consecutive to postsurgical malabsorption with pathologic fracture), M80.4 (drug-induced osteoporosis with pathologic fracture), M80.5 (idiopathic osteoporosis with pathologic fracture), M80.8 (other osteoporosis with pathologic fracture), M80.9 (osteoporosis, no further mention with pathologic fracture), M84.4 (pathologic fracture, not classified elsewhere).

- Category 2: osteoporosis-related clinical entities not defined as osteoporosis, including back pain and kyphosis. Comprises six relevant ICD-10 codes (Table 1).

- Category 3: fractures possibly due to osteoporosis, typically those caused by low-energy trauma. Comprises 28 eligible ICD-10 codes (Table 2).

- Category 4: fractures unlikely to be due to osteoporosis, typically those caused by high-energy trauma. Comprises 29 relevant ICD-10 codes: S42.0 (fracture clavicula), S42.4 (fracture humerus distal), S42.7 (multiple fractures clavicula), S42.8 (other

Table 1 Osteoporosis attribution rates by age group for osteoporosis-related clinical entities

\begin{tabular}{|c|c|c|c|c|c|c|c|}
\hline \multirow{2}{*}{$\frac{\text { Main diagnosis }}{\text { ICD-10 }}$} & \multirow[t]{2}{*}{ ICD-10 description } & \multicolumn{6}{|c|}{ Attribution rates by age group } \\
\hline & & $0-44$ & $45-54$ & $55-64$ & $65-74$ & $75-84$ & $85+$ \\
\hline M54.5 & Back pain & 0.00 & 0.25 & 0.38 & 0.50 & 0.50 & 0.50 \\
\hline M54.6 & Pain in thoracic spine & 0.00 & 0.40 & 0.49 & 0.58 & 0.58 & 0.58 \\
\hline M54.8 & Other back pain & 0.00 & 0.25 & 0.38 & 0.50 & 0.50 & 0.50 \\
\hline M54.9 & Back pain, without further mention & 0.00 & 0.25 & 0.38 & 0.50 & 0.50 & 0.50 \\
\hline M40.1 & Other secondary kyphosis & 0.00 & 0.85 & 0.85 & 0.85 & 0.85 & 0.85 \\
\hline M40.2 & $\begin{array}{l}\text { Other secondary kyphosis, with no } \\
\text { further mention }\end{array}$ & 0.00 & 0.85 & 0.85 & 0.85 & 0.85 & 0.85 \\
\hline
\end{tabular}


Table 2 Osteoporosis attribution rates by age group for fractures possibly due to osteoporosis

\begin{tabular}{|c|c|c|c|c|c|c|c|}
\hline \multirow{2}{*}{$\frac{\text { Main diagnosis }}{\text { ICD-10 }}$} & \multirow[t]{2}{*}{ ICD-10 description } & \multicolumn{6}{|c|}{ Attribution rates by age group } \\
\hline & & $0-44$ & $45-54$ & $55-64$ & $65-74$ & $75-84$ & $85+$ \\
\hline M48.4 & Vertebral fatigue fracture & 0.00 & 0.82 & 0.85 & 0.88 & 0.89 & 0.89 \\
\hline M48.5 & $\begin{array}{l}\text { Vertebral compression fracture, } \\
\text { not classified elsewhere }\end{array}$ & 0.00 & 0.72 & 0.74 & 0.75 & 0.75 & 0.75 \\
\hline M84.3 & Stress fracture, not classified elsewhere & 0.00 & 0.82 & 0.85 & 0.88 & 0.89 & 0.89 \\
\hline $\mathrm{S} 22.0$ & Fracture of the thoracic spine & 0.00 & 0.72 & 0.74 & 0.75 & 0.75 & 0.75 \\
\hline S22.1 & Multiple fractures of the thoracic spine & 0.00 & 0.72 & 0.74 & 0.75 & 0.75 & 0.75 \\
\hline S22.3 & Rib fracture & 0.00 & 0.72 & 0.74 & 0.75 & 0.75 & 0.75 \\
\hline S32.0 & Fracture of the lumbar spine & 0.00 & 0.60 & 0.68 & 0.75 & 0.90 & 0.90 \\
\hline S32.1 & Fracture of the sacrum & 0.00 & 0.60 & 0.68 & 0.75 & 0.90 & 0.90 \\
\hline S32.5 & Fracture of the pubis & 0.00 & 0.60 & 0.68 & 0.75 & 0.90 & 0.90 \\
\hline S32.7 & Multiple fractures of the lumbar spine & 0.00 & 0.60 & 0.68 & 0.75 & 0.90 & 0.90 \\
\hline S32.8 & Other fractures of the lumbar spine & 0.00 & 0.60 & 0.68 & 0.75 & 0.90 & 0.90 \\
\hline S42.2 & Fracture of the proximal humerus & 0.00 & 0.40 & 0.45 & 0.50 & 0.70 & 0.70 \\
\hline S42.3 & Fracture of the humerus shaft & 0.00 & 0.40 & 0.45 & 0.50 & 0.70 & 0.70 \\
\hline S52.2 & Fracture of the ulna shaft & 0.00 & 0.70 & 0.74 & 0.78 & 0.84 & 0.84 \\
\hline S52.5 & Fracture of the distal radius & 0.00 & 0.70 & 0.74 & 0.78 & 0.84 & 0.84 \\
\hline S52.6 & Combined fracture of the distal radius/ulna & 0.00 & 0.70 & 0.74 & 0.78 & 0.84 & 0.84 \\
\hline S72.0 & Fracture of the femoral neck & 0.00 & 0.51 & 0.61 & 0.71 & 0.91 & 0.91 \\
\hline S72.1 & Pertrochanteric fracture & 0.00 & 0.51 & 0.61 & 0.71 & 0.91 & 0.91 \\
\hline S72.2 & Subtrochanteric fracture & 0.00 & 0.51 & 0.61 & 0.71 & 0.91 & 0.91 \\
\hline S72.4 & Fracture of the femur, distal & 0.00 & 0.51 & 0.61 & 0.71 & 0.91 & 0.91 \\
\hline S72.8 & Fracture of the femur, other & 0.00 & 0.51 & 0.61 & 0.71 & 0.91 & 0.91 \\
\hline S72.9 & Fracture of the femur, no further mention & 0.00 & 0.51 & 0.61 & 0.71 & 0.91 & 0.91 \\
\hline S82.1 & Fracture of the tibia, proximal & 0.00 & 0.40 & 0.45 & 0.50 & 0.60 & 0.60 \\
\hline $\mathrm{S} 82.2$ & Fracture of the tibia shaft & 0.00 & 0.40 & 0.45 & 0.50 & 0.60 & 0.60 \\
\hline $\mathrm{S} 82.3$ & Fracture of the tibia, distal & 0.00 & 0.40 & 0.45 & 0.50 & 0.60 & 0.60 \\
\hline S82.4 & Fracture of the fibula & 0.00 & 0.40 & 0.45 & 0.50 & 0.60 & 0.60 \\
\hline $\mathrm{S} 82.5$ & Fracture of the malleolar int & 0.00 & 0.40 & 0.45 & 0.50 & 0.60 & 0.60 \\
\hline S82.6 & Fracture of the malleolar ext & 0.00 & 0.40 & 0.45 & 0.50 & 0.60 & 0.60 \\
\hline
\end{tabular}

fractures of the shoulder, upper arm), S42.9 (fracture of the shoulder, no further mention), S52.0 (fracture ulna proximal), S52.1 (fracture radius proximal), S52.3 (fracture of the radius shaft), S52.4 (combined fracture of the radius/ulna), S52.7 (multiple fractures of the forearm), S52.8 (fracture of the forearm, other), S52.9 (fracture of the forearm, no further mention), S62 (fracture of the hand and wrist), S72.3 (fracture of the femur shaft), S72.7 (multiple fractures femur), S82.0 (fracture patella), S82.7 (multiple fractures of the lower leg), S82.8 (fractures of the lower leg, other), S82.9 (fracture of the lower leg, no further mention), S92 (fracture of the foot, excluding ankle), T02.1 (fractures involving thoracic and lumbar spine and pelvis), T02.2 (multiple fractures of the upper limb), T02.3 (multiple fractures of the lower limb), T02.4 (multiple fractures of both upper limbs), T02.5 (multiple fractures of both lower limbs), T02.6 (multiple fractures of the lower and upper limbs), T02.7 (fractures involving thoracic and lumbar spine and pelvis and limbs), T02.8 (other combined fractures), T02.9 (multiple fractures, no further mention).

\section{Osteoporosis attribution rates}

The percentage of hospitalization cases related to osteoporosis was estimated by applying the best matching age-specific attribution rates published for the ICD-9 diagnosis codes $[7,8,9]$ to the individual ICD-10 codes. To preserve comparability with our previous publication, we used the conservative attribution rates published by Phillips et al. [8], although a more recent and detailed publication from Melton et al. examines agespecific and gender-specific attribution rates and indicates that osteoporosis might be the underlying cause of fracture more frequently than initially suspected [9]. In category 1 all recorded cases were counted as being due to osteoporosis (attribution rate 100\%). In category 2 all cases were weighted according to the age-specific attribution rates (Table 1). In category 3 (main diagnosis fracture), cases with a secondary diagnosis of osteoporosis were attributed to osteoporosis (attribution rate $100 \%$ ) and cases with a secondary diagnosis other than osteoporosis were weighted according to the agespecific attribution rates (Table 2). In category 4 (main diagnosis fracture), cases with a secondary diagnosis of osteoporosis were attributed to osteoporosis (attribution rate $100 \%$ ), while cases with a secondary diagnosis other than osteoporosis were considered as not being due to osteoporosis (attribution rate $0 \%$ ).

Data pooling for analysis

In a second step, to preserve comparability with previously published data [7], we grouped the ICD-10 frac- 
ture codes into seven diagnosis pools and tabulated them separately for women (Table 3) and men (Table 4) as follows: (1) fractures of the axial skeleton, (2) fractures of the proximal upper limbs, (3) fractures of the distal upper limbs, (4) fractures of the proximal lower limbs, (5) fractures of the distal lower limbs, (6) multiple fractures and (7) osteoporosis.

Calculation of the incidences of hospitalization due to fractures

The structure of the Swiss population in 2000 was used, divided into six age groups by gender $(0-44,45-54,55-$ $64,65-74,75-84$ and $\geq 85$ years), as published by the Swiss Federal Office of Statistics.

Estimation of the direct medical costs related to hospitalization

The Administrative Statistics showed the operational cost for general hospitals (including university hospitals) as being CHF 1,009 per hospital day and CHF 703 as the weighted average cost per day in special or rehabilitation clinics. It was assumed that within a given age group the mean length of stay was the same whether osteoporosis was the underlying cause or not. We multiplied the number of hospital days by the number of cases and by the cost per day to obtain an estimation of the total hospitalization costs related to osteoporosis and its complications.

Selected diseases used as comparators

Hospitalization days caused by COPD (ICD-10 codes J40-J47), stroke (I64, I66), acute myocardial infarction (I21, I22), heart failure (I50), diabetes (E10-E14) and breast carcinoma (C50) were assessed by the same methodology as described above. Those diseases represent well-established nosologic entities and are usually coded as the main diagnosis if they are the main reason for hospitalization. In contrast to osteoporosis, where fractures can be attributed to the underlying disease with a given probability, no such attribution rates exist for the complications of the selected comparator diseases. Therefore, we focused on the main diagnosis only.

\section{Results}

Hospitalizations due to fractures and osteoporotic fractures

In Switzerland 62,535 hospitalizations for fractures $(35,586$ women and 26,949 men) occurred during 2000 (Tables 3 and 4). Overall, $51 \%$ of those hospitalizations in women and $24 \%$ in men were considered to be due to osteoporosis, with large differences according to fracture site and gender. Thus, $80 \%$ of the hospitalizations for fractures of the proximal lower limbs and $59.3 \%$ of those for fractures of the axial skeleton were considered as osteoporotic in women $(56.5 \%$ and $37.5 \%$, respectively, in men), whereas $41 \%$ of the hospitalizations for distal upper limb fractures and $20 \%$ of those for distal lower limb fractures were considered as osteoporotic in women $(8.9 \%$ and $13.1 \%$, respectively, in men). In women, osteoporosis coded as such (ICD-10 M81 and M82) accounted for $3.5 \%$ (compared to $1.6 \%$ in men) of the total hospitalized cases attributable to osteoporosis based on the attribution rates.

Incidences of hospitalizations due to fractures

The incidences of hospitalization due to fractures were 969 and 768 per 100,000 in women (Table 3) and men (Table 4), respectively. Pooled incidences by fracture site and age group are shown for women and men in Figs. 1 and 2, respectively. The incidence of hospitalization for fractures of the proximal lower limbs and the axial skeleton sharply increased after the age of 65 years in both genders. The incidence of fractures of the proximal lower limbs was higher in men than in women up to the age of 54 years, and similar at 45-54 years $(108 / 100,000$ and $111 / 100,000$, respectively) and rose exponentially thereafter in both genders. At age $85+$ the incidence was $3,874 / 100,000$ in women and 1,940/100,000 in men. The incidence of hospitalization for fractures of the axial skeleton also increased strongly with age, from 68/ $100,000(0-44$ years $)$ to $1,690 / 100,000(85+$ years $)$ in women, and from $97 / 100,000$ to $784 / 100,000$ in $m e n$ in the same age groups.

\section{Direct medical costs related to hospitalization}

The total estimated cost due to osteoporosis and related fractures in Switzerland in 2000 was 357 million CHF.

In women, a total of 35,586 hospitalizations, amounting to 503,319 hospital days, were estimated to be due to fractures in 2000. The mean length of stay was 14.1 days. The total hospital costs were approximately 473 million CHF, corresponding to a mean cost per case of 13,312 CHF. In all, 18,037 hospitalizations have been estimated to be due to osteoporotic fractures and accounted for a total of 286,397 hospital days, with a mean length of stay of 15.9 days. The total hospital cost was estimated at 263.6 million $\mathrm{CHF}$, corresponding to a mean cost per case of $14,616 \mathrm{CHF}$. The hip fracture group had the largest number of cases and the longest length of stay (19.1 days, on average) and accounted for more than $50 \%$ of the estimated total hospitalization costs for osteoporotic fractures. Fractures of the axial skeleton accounted for $30 \%$ of these costs (Table 3 ).

In men, the number of hospitalizations due to fractures in general was $26^{\prime} 949$ in 2000 , with a mean length 


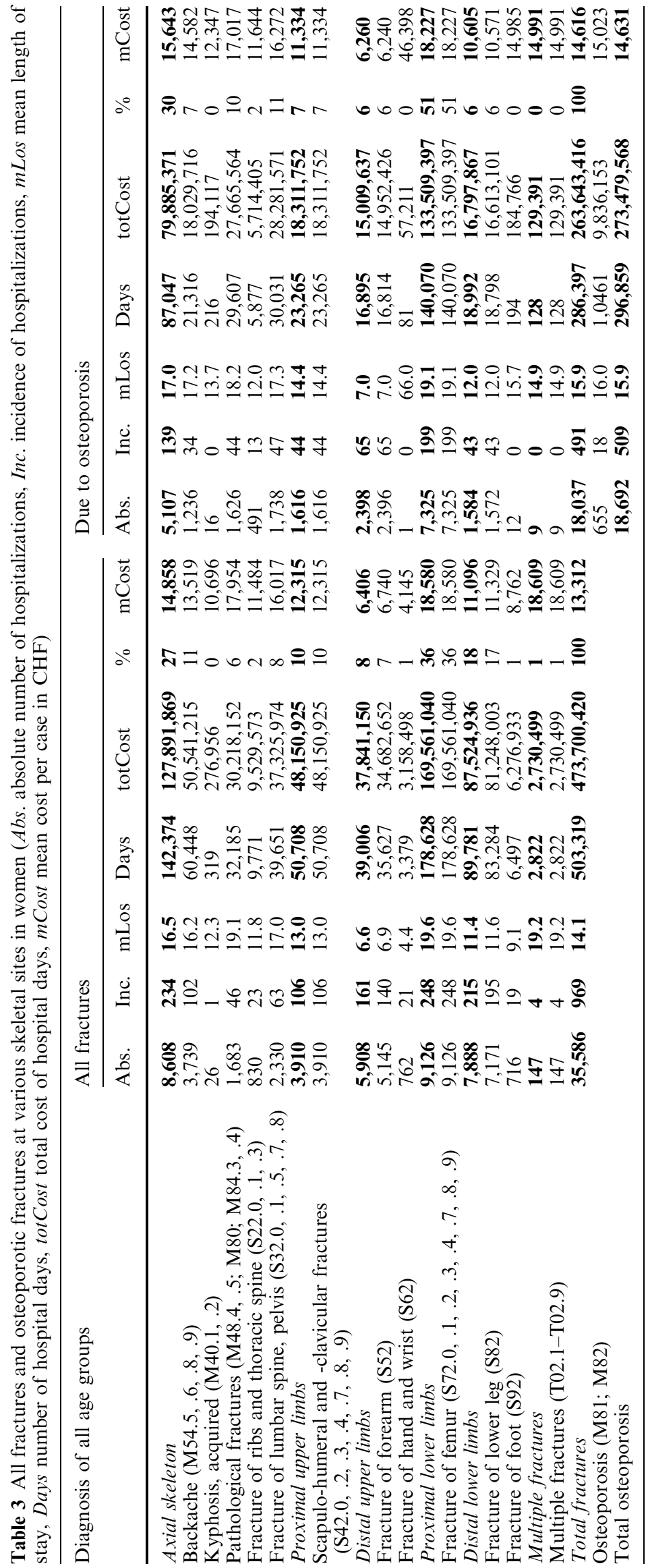




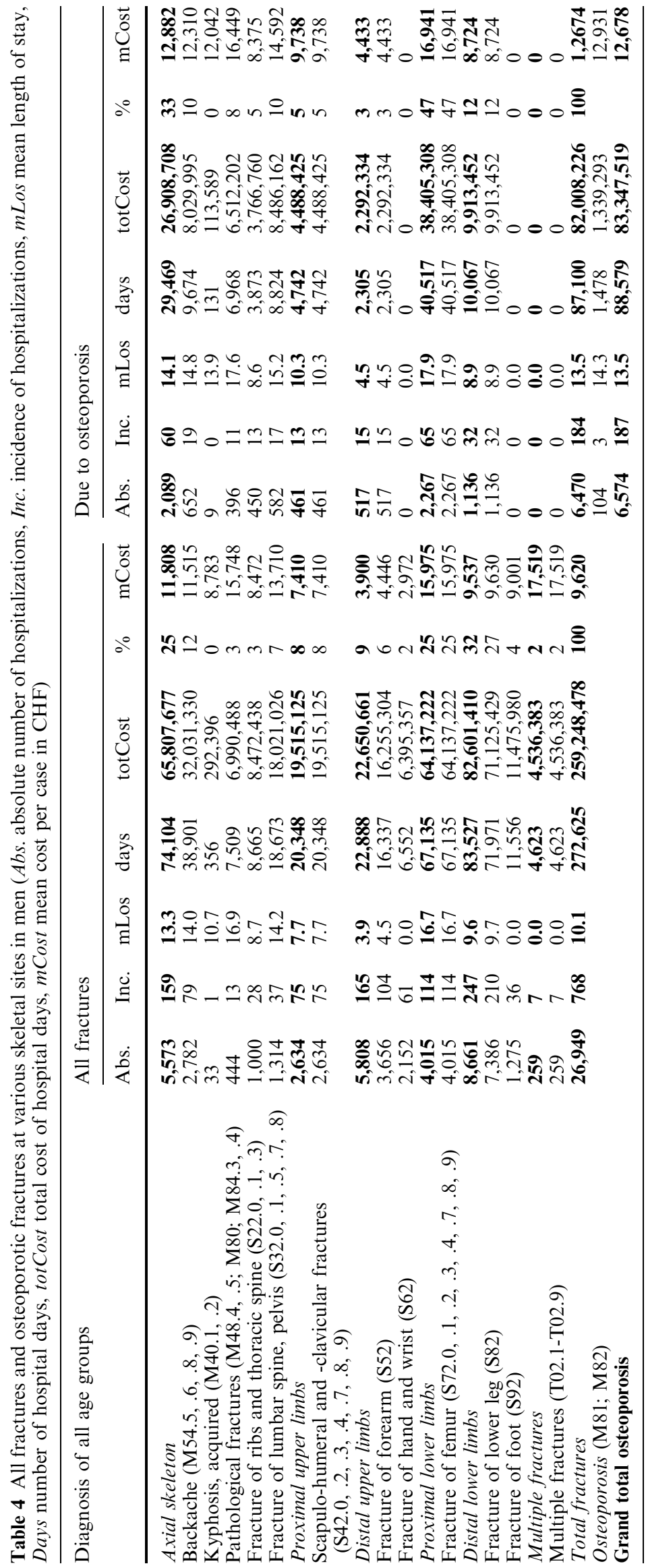




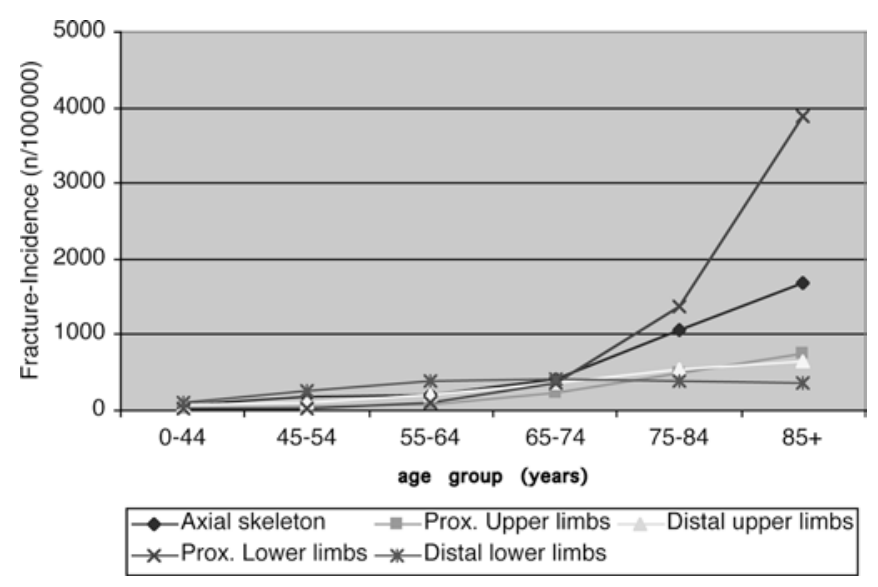

Fig. 1 Incidences of hospitalization due to fractures in Swiss women in year 2000 , by age group

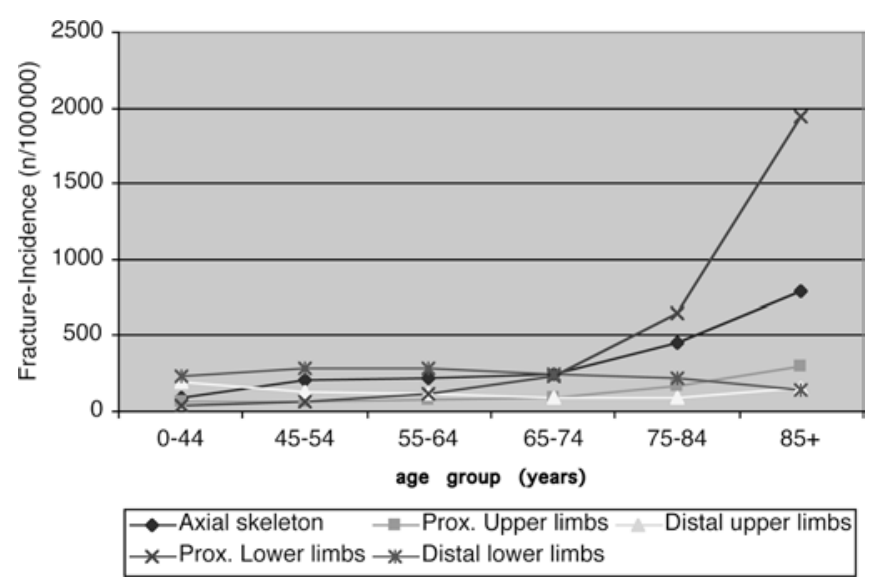

Fig. 2 Incidences of hospitalization due to fractures in Swiss men in year 2000 , by age group

of stay of 10.1 days, i.e., 4.0 days fewer or $28 \%$ less than in women. These cases accounted for 272,625 hospital days, i.e., almost $50 \%$ less than in women. The cost of hospitalizations was estimated at 259.2 million $\mathrm{CHF}$, corresponding to a mean cost per case of 9,620 CHF. Twenty-four percent of all hospitalizations for fractures were considered as related to osteoporosis, with 87,100 hospital days and 13.5 days average length of stay (LOS). The estimated cost of hospitalization due to osteoporotic fractures was 82 million $\mathrm{CHF}$, with a mean cost per case of 12,674 CHF. As in women, the main cost drivers were hip fractures. They were more frequent and produced the longest LOS (17.9 days), accounting for $47 \%$ of the estimated total hospitalization costs for osteoporotic fractures. Fractures of the axial skeleton accounted for $33 \%$ of these costs (Table 4 ).

\section{Comparison with other frequent diseases}

In women the age-independent incidence of hospitalization for osteoporosis or its complications was twice as

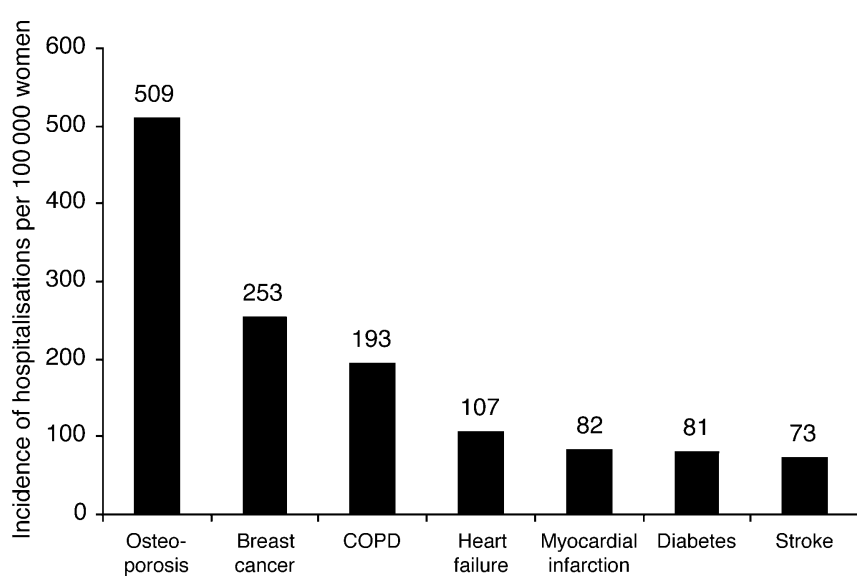

Fig. 3 Incidences of hospitalization for osteoporosis and other frequent diseases in Swiss women in year 2000

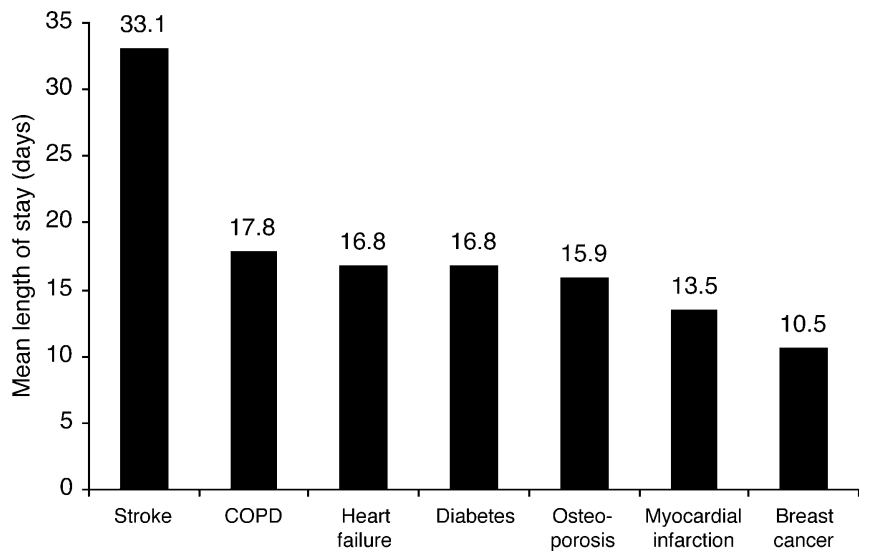

Fig. 4 Mean length of stay for osteoporosis and other frequent diseases in Swiss women in year 2000

high as that for breast cancer and pooled cardiovascular diseases (heart failure and myocardial infarction and stroke), almost three-times as high as that for COPD and six-times higher than that for diabetes (Fig. 3). The mean LOS for osteoporosis was similar to that of all the other diseases included in the analysis, with the exception of stroke (twice as long) and breast cancer $(50 \%$ shorter) (Fig. 4). Osteoporosis accounted for 1.5-times more hospital days than pooled cardiovascular diseases, two-to-three-times more than COPD or breast cancer and six-times more than diabetes (Fig. 5).

In men the disease with the highest hospitalization incidence and the highest number of hospital days was COPD. The overall incidence of hospitalizations for osteoporosis was identical to the incidence of hospitalizations for myocardial infarction, with a longer mean length of stay and almost $50 \%$ more hospital days (Figs. 6, 7 and 8).

\section{Discussion}

In a previous study [7], which used similar methods to analyze the 1992 statistics database of the VESKA 


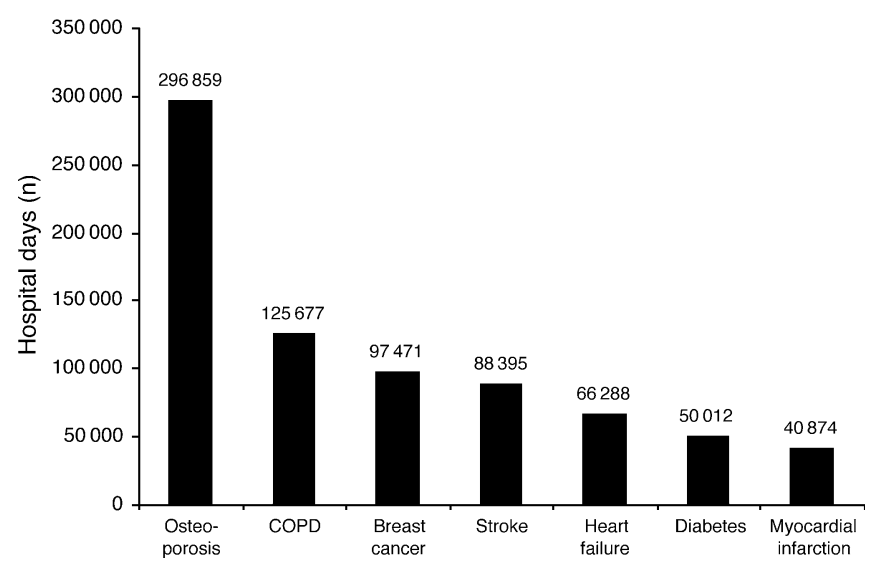

Fig. 5 Hospital days for osteoporosis and other frequent diseases in Swiss women in year 2000

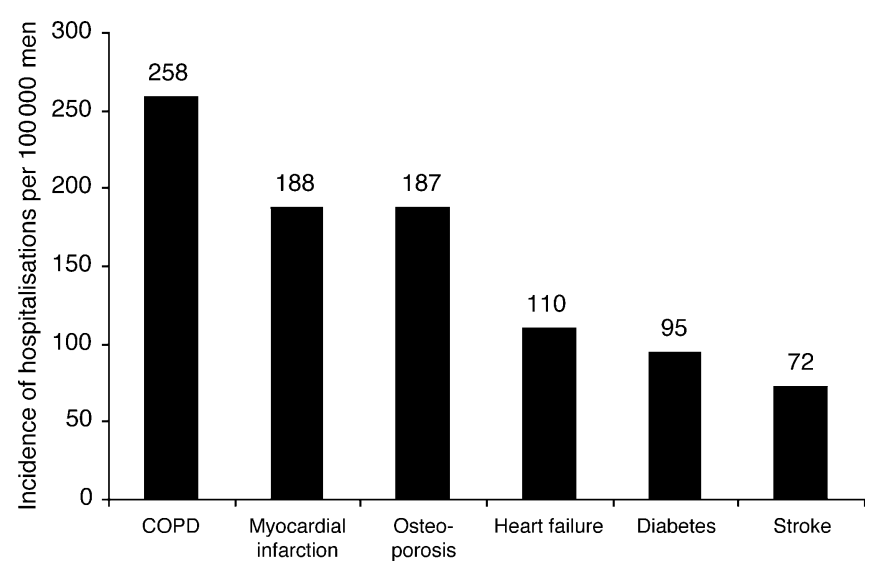

Fig. 6 Incidences of hospitalization for osteoporosis and other frequent diseases in Swiss men in year 2000

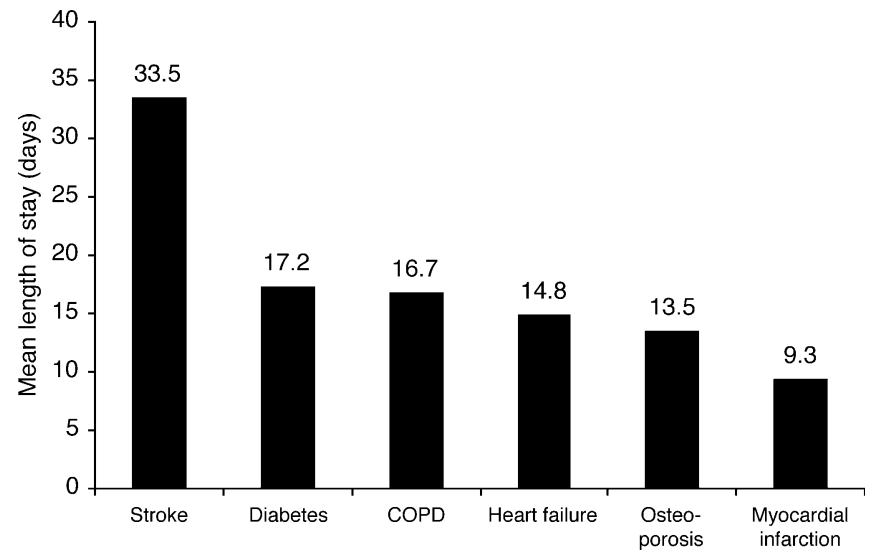

Fig. 7 Mean length of stay for osteoporosis and other frequent diseases in Swiss men in year 2000

(Vereinigung Schweizer Krankenhäuser, Federation of Swiss Hospitals), we assessed the incidence rate of fractures and estimated the direct medical costs linked to hospitalizations for osteoporosis and its complications.

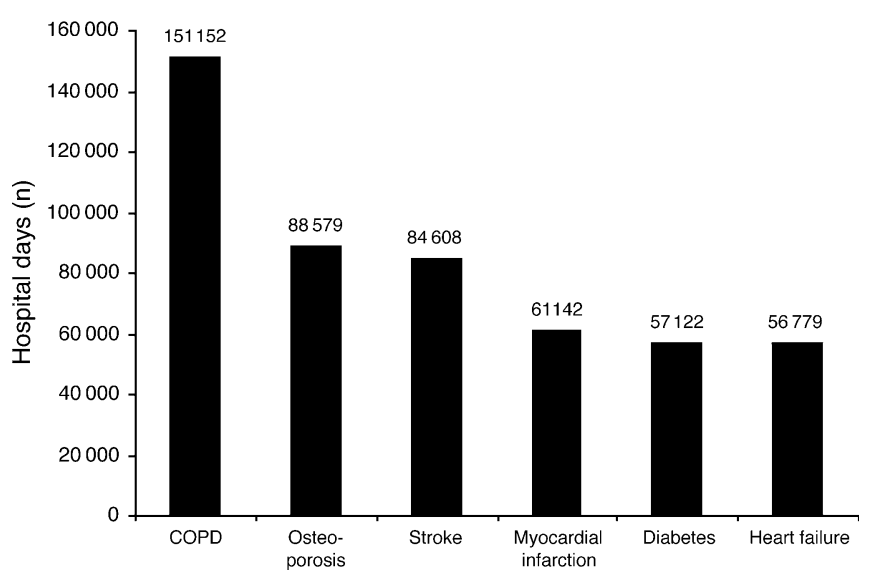

Fig. 8 Hospital days for osteoporosis and other frequent diseases in Swiss men in year 2000

The 1992 data showed a total of 63,170 hospitalizations for fractures (53\% in women) and the annual incidences of hospitalizations for fractures were 950 per 100,000 and 877 per 100,000 in women and men, respectively. In 2000 these values were very similar: 62,535 hospitalizations for fractures, $57 \%$ in women, and incidences of 969 per 100,000 and 768 per 100,000 in women and men, respectively. In 1992 the total number of hospitalized osteoporotic fractures was 18,380 in women (vs 18,037 in 2000) and 7,619 in men (vs 6,470 in 2000). Between 1992 and 2000 the average length of stay shortened considerably, from 24.3 days to 15.9 days (-8.4 days) in the case of women and from 18.2 to 13.5 days ( -4.7 days) in the case of men. This led to a decrease of almost $40 \%$ in direct medical costs related to acute hospitalizations for osteoporosis.

As compared to 1992, the number of osteoporotic fractures of the proximal lower limbs (mainly hip fractures) increased by almost $10 \%$ in women and $21 \%$ in men. In women the number of hospitalizations due to fractures of the axial skeleton increased by $25 \%$. For all other fracture sites the number of hospitalizations decreased by up to $35 \%$ (Table 5). The lengths of stay decreased markedly for all fracture types, the largest change being seen for hip fractures (around 50\%) and the least change for fractures of the axial skeleton (around 25\%), (Table 6). In women in 1992 osteoporosis was the leading disease, followed by COPD, stroke, breast cancer and myocardial infarction. This ranking, and the respective orders of magnitude, remained similar in 2000. In men in 1992 COPD was the leading cause of hospitalization, followed by myocardial infarction, stroke and osteoporosis. In 2000, in men, osteoporosis progressed from rank 4 to rank 2, ahead of stroke and myocardial infarction.

There are several possible explanations for these observations. The Swiss statistical data collection system has recently changed: in 1992 the VESKA statistics were derived from a representative sample of $43 \%$ of the Swiss hospitals, based on voluntary membership, which we extrapolated to $100 \%$. In 2000 , membership of the 
Table 5 Number of hospitalizations for osteoporotic fractures, year 2000 compared to 1992

\begin{tabular}{llll}
\hline Fracture & $\begin{array}{l}\text { Hospitalizations } \\
1992\end{array}$ & 2000 & $\begin{array}{l}\text { Difference } \\
\text { 2000 vs } 1992\end{array}$ \\
\hline Women & & & \\
Proximal lower limbs & 6,690 & 7,325 & $+9.5 \%$ \\
Distal upper limbs & 3,449 & 2,398 & $-30.5 \%$ \\
Proximal upper limbs & 1,885 & 1,616 & $-14.3 \%$ \\
Axial skeleton & 4,092 & 5,107 & $+24.8 \%$ \\
Distal lower limbs & 2,264 & 1,584 & $-30.0 \%$ \\
Men & & & \\
Proximal lower limbs & 1,876 & 2,267 & $+20.8 \%$ \\
Distal upper limbs & 989 & 517 & $-47.7 \%$ \\
Proximal upper limbs & 708 & 461 & $-34.9 \%$ \\
Axial skeleton & 2,669 & 2,089 & $-21.7 \%$ \\
Distal lower limbs & 1,377 & 1,136 & $-17.5 \%$ \\
\hline
\end{tabular}

Table 6 Mean length of hospital stay for osteoporotic fractures, 2000 compared to 1992

\begin{tabular}{llll}
\hline Fracture & $\begin{array}{l}\text { Length of stay 1992 } \\
\text { (days) }\end{array}$ & $\begin{array}{l}\text { Length of stay 2000 } \\
\text { (days) }\end{array}$ & Difference 2000 vs 1992 \\
\hline Women & & & \\
$\quad$ Proximal lower limbs & 30.8 & 19.1 & $-38.0 \%$ \\
$\quad$ Distal upper limbs & 11.0 & 7.0 & $-36.4 \%$ \\
Proximal upper limbs & 30.5 & 14.4 & $-52.8 \%$ \\
Axial skeleton & 24.5 & 17.0 & $-30.6 \%$ \\
Distal lower limbs & 19.6 & 12.0 & $-38.8 \%$ \\
Men & 24.5 & 17.9 & $-26.9 \%$ \\
Proximal lower limbs & 9.9 & 4.5 & $-54.6 \%$ \\
Distal upper limbs & 10.3 & $-45.2 \%$ \\
Proximal upper limbs & 18.8 & 14.1 & $-19.0 \%$ \\
Axial skeleton & 17.4 & 8.9 & $-47.0 \%$ \\
Distal lower limbs & 16.8 & & \\
\hline
\end{tabular}

SFOS was mandatory, and the data shown here were extrapolated from a statistics database representing $81.2 \%$ of all hospital admissions. The differences in source data and in the extrapolated sample size might have introduced some variance. However, as the 1992 VESKA data were a representative sample for all Swiss hospitals, and as a greater percentage of hospitals reported data in 2000 , the data shown here can be considered a realistic scenario of the number of fractures and hospitalizations in Switzerland in both years. The ICD coding system was changed from ICD-9 in 1992 to the more detailed ICD-10 in 2000. However, the ICD-10 contains codes similar to those in ICD-9, and the data pooling was done on the basis of diagnoses compatible with the ICD-9 data classification of 1992. In addition, the incidence of hip fractures, which almost always cause hospitalization, increased between 1992 and 2000, which is in line with long-term projections for several countries: an increase of $38 \%$ in hip fracture incidence between 1983 and 2010 was projected for Finland [10] and $45 \%$ between 1986 and 2020 in the USA [11]. Some scenarios even show an increase in the age-specific fracture risk, i.e., an increase in the osteoporosis attribution rate for hip fractures $[12,13]$. This has led to a prediction of a $150 \%$ increase in hip fracture rate in the UK between 1985 and 2016 [14]. For Switzerland, based on the population ageing scenarios predicted by the SFOS, and assuming that the osteoporosis attribution rate by age group would remain constant, we estimated that an increase in the annual incidence of hip fracture cases, by 55\% between 1992 and 2020, could be realistically expected [15].

The large decrease in the mean length of stay observed for all fractures may have several explanations. Cost containment measures have affected the Swiss healthcare system increasingly over the past 10 years, leading to cost savings at the hospital level. These savings have been brought about by a shift from hospitalization to ambulatory treatment and by earlier hospital discharge as a result of improved orthopedic surgical techniques and the shift to ambulatory post-surgery follow-up. A similar decrease in length of stay has been observed with all the other common diseases used as comparators. In women the average LOS for breast cancer hospitalizations was reduced from 25.7 days in 1992 to 10.5 days in 2000 . A similar reduction was observed for heart failure (from 27.1 to 16.8 days), diabetes (from 25.2 to 15.9 days), myocardial infarction (from 16.7 to 13.5 days), stroke (from 39.9 to 33.1 days) and COPD (from 20.1 to 17.8 days). In men the average LOS was reduced for all the diseases, with the exception of stroke, used as comparators (myocardial infarction decreased from 14.1 to 9.3 days, heart failure from 18.1 to 14.8 days, diabetes from 19.5 to 17.2 days, COPD from 17.7 to 16.7 days; stroke increased from 30.0 to 33.5 days).

It is, therefore, likely that a major part of the direct medical costs of osteoporosis have moved from the well- 
measurable hospital segment to the ambulatory segment, for which we do not yet have the assessment and measurement tools in Switzerland.

Since 1996, drugs with a proven effect on the individual fracture risk at all clinically relevant sites, such as the bisphosphonates alendronate and risedronate, have been available to Swiss physicians. Although these drugs prevent hip fractures (significant reduction of fracture risk up to $50 \%$ in well-designed fracture endpoint trials, with a measurable between-groups difference after 36 months $[16,17,18,19])$, and although the efficacy of vitamin D substitution and nutritional measures in institutionalized elderly people is well documented [20, $21,22]$, there has been an increase in hospitalizations for hip fractures over the past 8 years. Projections from bisphosphonate sales data (IHA/IMS Health, Hergiswil, 2003 ) for 2000 suggest that fewer than $10 \%$ of Swiss women with osteoporosis were being treated with these drugs. The year 2000 fracture and hospitalization data might, therefore, fail to detect a treatment effect on fracture risk. According to the same source, the availability of the new once-weekly formulations would broaden the use of these drugs to approximately $20 \%$ of all women with osteoporosis by the end of 2003, making it more likely that we will observe a population effect in the future. However, the awareness of osteoporosis remains low in Switzerland. In the present analysis, and in the 9,126 reported cases of femoral fracture, only 346 $(5.4 \%)$ mentioned osteoporosis as a secondary diagnosis in women. This value drops to $1.9 \%$ in men.

Osteoporosis and its complications were the leading drivers of direct medical costs related to hospitalization in women in 2000 in Switzerland and were comparable, as cost drivers, to myocardial infarction in men. Although progress has been made in shortening acute hospitalization stays and ambulatory treatment of fractures, the costs related to osteoporosis have been shifted into the ambulatory cost segment, for which the assessment and management tools remain underdeveloped. Awareness of the consequences of the disease remains low. This prevents the widespread use of drugs that decrease fracture risk in osteoporotic patients and, thereby, limits their potential contribution to cost containment.

Acknowledgements This study was supported by an unrestricted educational grant from Aventis Pharma AG Switzerland. We thank Dr. Philippe Kress for his invaluable contribution to the manuscript.

\section{References}

1. Anonymous (1993) Consensus development conference. Diagnosis, prophylaxis and treatment of osteoporosis. Am J Med 94:646-650
2. Melton LJ III (1988) Epidemiology of fractures. In: Riggs BL, Melton LJ (eds) Osteoporosis: etiology, diagnosis and management. Raven Press, New York, pp 133-155

3. Nilsson BE (1969) Age and incidence of ankle fractures. Acta Orthop Scand 40:122-129

4. Melton LJ III, Sampson JM, Morrey BF, Illstrup DM (1981) Epidemiologic features of pelvic fractures. Clin Orthop 155:4347

5. Rose SH, Melton LJ III, Morrey BF, Illstrup DM, Riggs BL (1982) Epidemiologic features of humeral fractures. Clin Orthop 168:24-30

6. Seeley DG, Browner WS, Nevitt MC, Genant HK, Scott JC, Cummings SR (1991) Which fractures are associated with low appendicular bone mass in elderly women? Ann Intern Med 115:837-842

7. Lippuner K, von Overbeck J, Perrelet R, Bosshard H, Jaeger P (1997) Incidence and direct medical costs of hospitalizations due to osteoporotic fractures in Switzerland. Osteoporos Int 7:414-425

8. Phillips S, Fox N, Jacobs J, Wright WE (1988) The direct medical cost of osteoporosis for American women aged 45 and older, 1986. Bone 9:271-279

9. Melton LJ III, Thamer M, Ray NF, et al (1997) Fractures attributable to osteoporosis: report from the National Osteoporosis Foundation. J Bone Miner Res 12:16-23

10. Simonen O (1988) Epidemiology and socio-economic aspects of osteoporosis in Finland. Ann Chir Gynaecol 77:173-175

11. Cummings SR, Rubin SM, Black D (1990) The future of hip fractures in the United States. Numbers, costs, and potential effects of postmenopausal estrogen. Clin Orthop 252:163-166

12. Melton LJ III, O'Fallon WM, Riggs BL (1987) Secular trends in the incidence of hip fractures. Calcif Tissue Int 41:57-64

13. Melton LJ III (1993) Hip fractures: a worldwide problem today and tomorrow. Bone 14 [Suppl 1]:S1-8

14. Hoffenberg R, James OFW, Brocklehurst JC, et al (1989) Fractured neck of the femur: prevention and management: summary and recommendations of a report of the Royal College of Physicians. J R Coll Physicians Lond 23:8-12

15. Lippuner K, Jaeger P (1998) Direkte Spitalkosten durch osteoporosebedingte Hüftfrakturen in der Schweiz heute und im Jahre 2020. Ein sozio-ökonomischer Alptraum. Schweiz Aerztezeitung 79:690-697

16. Black DM, Thompson D, Bauer DC, Ensrud K, et al. (2000) Fracture risk reduction with alendronate in women with osteoporosis: the Fracture Intervention Trial. J Clin Endocrinol Metab 85:4118-4124

17. Black, DM, Cummings SR, Karpf DB, et al (1996) Randomised trial of effect of alendronate on risk of fracture in women with existing vertebral fractures. Lancet 348:1535-1541

18. Cummings SR, Black DM, Thompson DE, et al. (1998) Effect of alendronate on risk of fracture in women with low bone density but without vertebral fractures: results from the Fracture Intervention Trial. JAMA 280:2077-2082

19. McClung MR, Geusens P, Miller PD, et al (2001) Effect of risedronate on the risk of hip fracture in elderly women. N Engl J Med 344:333-340

20. Chapuy MC, Arlot ME, Delmas PD, Meunier PJ (1994) Effect of calcium and cholecalciferol treatment for three years on hip fractures in elderly women. BMJ 308:1081-1082

21. Chapuy MC, Arlot ME, Duboeuf F, et al (1992) Vitamin $D_{3}$ and calcium to prevent hip fractures in elderly women. N Engl J Med 327:1637-1642

22. Chevalley T, Rizzoli R, Nydegger V, et al (1994) Effects of calcium supplements on femoral bone mineral density and vertebral fracture rate in vitamin-D-replete elderly patients. Osteoporos Int 4:245-252 